SU-ITP-93-22

SSCL-Preprint-506-93

September, 1993

\title{
LOW ENERGY GAUGE COUPLINGS IN GRAND UNIFIED THEORIES AND HIGH PRECISION PHYSICS
}

\author{
BRYAN W L LYN \\ Department of Physics, Stanford University \\ Stanford, California 94305 \\ and \\ Superconducting Super Collider Laboratory \\ MS 2007, 2550 Beckleymeade, Dallas, Texas 75237-3997
}

Submitted to Physics Letters $B$ 


\section{DISCLAIMER}

Portions of this document may be illegible in electronic image products. Images are produced from the best available original document. 


\section{ABSTRACT}

I generalize the leading log relations between low energy $S U(3)_{Q C D}, S U(2)_{\vec{I}}$ and $U(1)_{Y}$ effective gauge couplings to include all one-loop threshold effects of matter fields in oblique vector self energy quantum corrections for both supersymmetric and non-supersymmetric SU(5) grand unified theories. These always involve an exactly conserved current from the unbroken $S U(3)_{Q C D} \times U(1)_{Q E D}$ subgroup; this fact strongly constrains any non-decoupling of heavy states as well as the generic character of threshold effects. Relations between low energy gauge couplings depend on the details of the spectra of both the superheavy $\left(M>10^{8}\right.$ $\mathrm{GeV})$ and low mass $(m<10 \mathrm{TeV})$ sectors; I display the common origin of the logs appropriate to superheavy matter states, which can be found with well known renormalization group techniques, and the combination of logs and polynomials (and worse) appropriate for light matter states, which cannot. Relations between any two or all three low energy effective gauge couplings do not depend on the top quark or standard model Higgs' masses. Neither do they depend on neutral color singlet states such as other neutral color singlet Higgs' or higgsinos, sneutrinos, zinos or photinos. Further, they do not depend on degenerate SU(5) matter representations, of either spin 0 or spin $\frac{1}{2}$, of any mass; matter representations of $\mathrm{SU}(5)$ can affect such relations only if there is mass splitting within them. The $b$ quark splitting from the $\tau$ and $\nu_{\tau}$ can affect the relation between gauge couplings for $\left|q^{2}\right| \sim m_{b}^{2}$ as can hadronic resonances and multi-hadron states for lower $\left|q^{2}\right|$. New mass-split representations of light states, such as occur in supersymmetric theories, can also affect such relations. A certain class of minimal supersymmetric SU(5) grand unified theories generically contains new light matter states $m \sim M_{Z}$; $\log$ and polynomial threshold effects may well play an important role in the comparison of the precise predictions of such supersymmetric grand unified theories with high precision experimental measurements. The results here also hold for any larger grand unified gauge group $\mathcal{G} \supseteq S U(5) \supset S U(3)_{Q C D} \times S U(2)_{\vec{I}} \times U(1)_{Y}$. 
Grand unified theories, especially supersymmetric theories ${ }^{[1][2]}$, have recieved a great deal of attention concerning their low energy predictions for fermion masses and mixings and, at the leading log level, their predictions for high precision relations between low energy gauge couplings ${ }^{\left[1{ }^{[3][4]}\right.}$. The first step in any high precision comparison of the predictions of theory with the results of experiment is the identification of exactly which precisely calculable theoretical quantity has actually been measured in the realistic experimental setup; figuratively, the comparison must be of apples to apples, oranges to oranges. Real experimental data involves complicated experimental cuts (defined by the geometry, electronics, etc. of the detector) and certain aspects of data acquisition (such as data triggering) as well as format simplifications (such as the removal of QED bremstrahlung) in the display of the published data. To say the least, meaningful comparison to theoretical predictions must be done very carefully. For high precision electroweak physics, this problem has been solved, at least for accuracies corresponding to fractions of a percent ${ }^{[5]}$. Apart from vertex, box, bremstrahlung and one particle irreducible two-loop contributions, high precision electroweak experiments measure the quantities entering into the "improved Born aproximation" ${ }^{[5]}$ of the low energy scattering and annihilation of massless fermions in four-fermion processes. In particular, experiments done at low momentum transfer $\left|q^{2}\right| \ll 1 \mathrm{TeV}^{2}$ measure the effective gauge couplings $g_{1}^{*}\left(q^{2}\right), g_{2}^{*}\left(q^{2}\right)$ and $g_{3}^{*}\left(q^{2}\right)$ corresponding to the low energy gauge groups $U(1)_{Y}, S U(2)_{\vec{I}}$ and $S U(3)_{Q C D}$ where the hypercharge $Y$ and third component of weak isospin $I_{3}$ are related to the electromagnetic charge; $Q=I_{3}+\frac{1}{2} Y$. The bare and effective couplings of the respective subgroups are related by ${ }^{[5]}$

$$
\begin{aligned}
& \frac{1}{\left(g_{1}^{*}\left(q^{2}\right)\right)^{2}}=\frac{1}{\left(g_{1}^{\text {bare }}\right)^{2}}-\Re \Pi_{Y Q}^{\prime}\left(q^{2}\right) \\
& \frac{1}{\left(g_{2}^{*}\left(q^{2}\right)\right)^{2}}=\frac{1}{\left(g_{2}^{\text {bare }}\right)^{2}}-\Re \Pi_{3 Q}^{\prime}\left(q^{2}\right) \\
& \frac{1}{\left(g_{3}^{*}\left(q^{2}\right)\right)^{2}}=\frac{1}{\left(g_{3}^{\text {bare }}\right)^{2}}-\Re \Pi_{g g}^{\prime}\left(q^{2}\right)
\end{aligned}
$$

where $3, Q, Y$, and $g$ indicate $I_{3}$, electric charge, $\frac{1}{2} Y$ hypercharge and color 
matter currents respectively. The $\Pi$ 's include only matter fields; these are to be carefully distinguished from gauge bosons, Goldstone bosons and ghosts. The real and imaginary parts of analytic functions are denoted by $\Re$ and $\Im$. A detailed account of the oblique ${ }^{[6][5]}$ vector particle self energy functions $\Pi_{i j}\left(q^{2}\right)$, along with the integrals appearing in them can be found elsewhere ${ }^{[7][6]} g_{i}^{\text {bare }}$ with $i=1,2,3$ is the appropriate bare gauge coupling constant appearing in the bare Lagrangian of the $S U(3) \times S U(2) \times U(1)$ low energy theory and also in the bare grand unified theory. The notation $g_{i}^{*}=g_{i}^{*}\left(q^{2}\right)$ is used for the effective running couplings where the $*$ indicates a function of the appropriate momentum transfer $q^{2}$. A crucial observation is that the vector boson self-energies appearing in the renormalization of gauge couplings contain at least one exactly conserved matter current from the unbroken subgroup $S U(3)_{Q C D} \times U(1)_{Q E D}$ and so can be written $\Pi_{\mu \nu}=\left(q^{2} \delta_{\mu \nu}-\right.$ $\left.q_{\mu} q_{\nu}\right) \Pi^{\prime}\left(q^{2}\right)$. The three $\Pi^{\prime}$ functions then include $\underline{\text { all }}$ one loop threshold effects from matter fields: logs, polynomials, everything.

Now write the leading log renormalization group relation between the gauge couplings of the $S U(3), S U(2)$ and $U(1)_{Y}$ subgroups of minimal supersymmetric $\mathrm{SU}(5)$, usually written $\sin ^{2} \theta \approx \frac{1}{5}+\frac{7}{15} \frac{\alpha_{Q E D}}{\alpha_{Q C D}}$ (and satisfied by LEP data), instead in the more revealing way $\left[\frac{1}{g_{1}^{2}}-4 \frac{1}{g_{2}^{2}}+\frac{7}{3} \frac{1}{g_{3}^{2}}\right]_{\text {leading } \ln \frac{M_{G U T}^{2}}{q^{2}}}=0$ where $\left|q^{2}\right| \ll 1 \mathrm{TeV}$ and the cancellation of the huge logs involving $q^{2}, m_{S U S Y}^{2}<(10 \mathrm{TeV})^{2}$ or $M_{W}^{2}$ and the grand unified scale $M_{G U T}^{2}$ is explicit. This is clearly nothing more than the leading log approximation to the exact relation

$$
\frac{1}{\left(g_{1}^{\text {bare }}\right)^{2}}-4 \frac{1}{\left(g_{2}^{\text {bare }}\right)^{2}}+\frac{7}{3} \frac{1}{\left(g_{3}^{\text {bare }}\right)^{2}}=0
$$

which is enforced by the symmetry and Clebsch-Gordon coefficients of $S U(5)$. The generalization of the leading log relation to include all oblique one loop threshold effects of matter fields consists only of rewriting (2)in terms of the experimentally measured effective gauge couplings $g_{i}^{*}$ using (1):

$$
\left[\left\{\frac{1}{\left(g_{1}^{*}\right)^{2}}+\Re \Pi_{Y Q}^{\prime}\right\}-4\left\{\frac{1}{\left(g_{2}^{*}\right)^{2}}+\Re \Pi_{3 Q}^{\prime}\right\}+\frac{7}{3}\left\{\frac{1}{\left(g_{3}^{*}\right)^{2}}+\Re \Pi_{g g}^{\prime}\right\}\right]_{q^{2}}=0
$$


All one loop threshold information about matter fields contained in the relation between the three low energy gauge couplings is therefore stored in a single function $\Delta_{S U_{5}}$ if I write (3)in the form

$$
\begin{aligned}
& \frac{1}{5}-s_{*}^{2}+\frac{7}{15} \frac{\alpha_{Q E D}^{*}}{\alpha_{Q C D}^{*}}+\frac{\alpha_{Q E D}^{*}}{20} \Delta_{S U_{5}}=0 \\
& \Delta_{S U_{5}}\left(q^{2}\right)=16 \pi \Re\left[\Pi_{Y Q}^{\prime}-4 \Pi_{3 Q}^{\prime}+\frac{7}{3} \Pi_{g g}^{\prime}\right]
\end{aligned}
$$

Here the usual notation for the electromagnetic coupling $\frac{1}{\left(g_{1}^{*}\right)^{2}}+\frac{1}{\left(g_{2}^{*}\right)^{2}}=\frac{1}{e_{*}^{2}}$ and the sine-squared of the weak mixing angle $s_{*}^{2}=\frac{e_{*}^{2}}{\left(g_{2}^{*}\right)^{2}}$ is used with $\alpha_{Q E D}^{*}=\frac{e_{*}^{2}}{4 \pi}$ and $\alpha_{Q C D}^{*}=\frac{\left(g_{3}^{*}\right)^{2}}{4 \pi}$ all functions, along with the $\Pi$ 's, evaluated at the experimental momentum transfer $q^{2} ; s_{*}^{2}, \alpha_{Q C D}^{*}$ and $\alpha_{Q E D}^{*}$ are then the experimentally measured quantities. The expressions (3)and (4)include the Dyson resummation $\Sigma_{n=0}^{n=\infty}(1 \text { loop })^{n}$ and so automatically resum leading logs. More general than the supersymmetric $\mathrm{SU}(5)$ starting point, they are correct for an arbitrary $\mathrm{SU}(5)$ grand unified theory with an arbitrary set of SU(5) matter representations.

The combination of oblique vector self energies appropriate to SU(5) must be ultraviolet finite for any $\mathrm{SU}(5)$ matter representation; it is not sufficient that it be finite for just supersymmetric $\mathrm{SU}(5)$. To see this, one need only remember that supersymmetric $\mathrm{SU}(5)$ is just a special case of $\mathrm{SU}(5)$; the process of supersymmetrization consists only of choosing specific spin 0 and spin $\frac{1}{2} \mathrm{SU}(5)$ matter representations with careful matching of the $\mathrm{SU}(5)$ quantum numbers of $R$ parity $R=0$ and $R \neq 0$ states. Indeed, the combination $\Delta_{S U_{5}}$ is ultraviolet finite for

\footnotetext{
* Although discussion of this point is beyond the scope of this paper, care has been taken here for quantum loops involving gauge bosons, Goldstone bosons and ghosts, to include certain "universal" vertex and box parts in order to cancel gauge dependent pole contributions in vector self energies and yield gauge invariant results ${ }^{[5]}$. When this is done, the huge logs $\sim \ln \frac{M_{G U T}^{2}}{M_{W}^{2}} \approx 60$ cancel in (3)and (4)for minimal supersymmetric SU(5). Such logs may reappear for arbitrary SU(5) Higgs' sectors or in the presence of new large heirarchies; it would then be more interesting to study a different combination of the exact equations $\frac{1}{\left(g_{1}^{b a r c}\right)^{2}}-\frac{5}{3} \frac{1}{\left(g_{2}^{\text {barc }}\right)^{2}}=0$ and $\frac{1}{\left(g_{2}^{\text {berc }}\right)^{2}}-\frac{1}{\left(g_{3}^{\text {bere }}\right)^{2}}=0$ where such logs in most part cancel.
} 
both supersymmetric and non-supersymmetric SU(5) matter representations. ${ }^{\dagger}$

Equally revealing are the decoupling and non-decoupling properties of $\Delta_{S U_{5}}$. These are profoundly constrained by the central result of (3)and (4); the oblique vector self energies appropriate to the relation between the three low energy gauge couplings contain at most one spontaneously broken current and at least one current from the unbroken $S U(3)_{Q C D} \times U(1)_{Q E D}$ subgroup. One consequence of this lies in the change in the relation between the three effective low energy gauge couplings as the experimental momentum transfer $q^{2}$ changes from spacelike $1 \mathrm{GeV}^{2}$ to the timelike $Z$ pole:

$$
\begin{aligned}
& {\left[\frac{1}{\left(g_{1}^{*}\right)^{2}}-4 \frac{1}{\left(g_{2}^{*}\right)^{2}}+\frac{7}{3} \frac{1}{\left(g_{3}^{*}\right)^{2}}\right]_{\left(q^{2}=-M_{Z}^{2}\right)}-\left[\frac{1}{\left(g_{1}^{*}\right)^{2}}-4 \frac{1}{\left(g_{2}^{*}\right)^{2}}+\frac{7}{3} \frac{1}{\left(g_{3}^{*}\right)^{2}}\right]_{\left(q^{2}=1 G e V^{2}\right)}} \\
& =\frac{1}{16 \pi}\left\{-\Delta_{S U_{5}}\left(q^{2}=-M_{Z}^{2}\right)+\Delta_{S U_{5}}\left(q^{2}=1 G e V^{2}\right)\right\}
\end{aligned}
$$

Equation (5)is protected by exact global symmetries from heavy states and therefore obeys a decoupling theorem; $\mathrm{SU}(5)$ matter representations, all of whose members are very heavy compared to the $Z$ mass, $M_{i^{\text {th }}}$ member of $S U_{5}$ representation $\gg$ $M_{Z}$, decouple from the running of all three low energy gauge couplings between $1 \mathrm{GeV}$ and the $Z$ mass. This is true even if there are global $S U(2)_{L+R}$ breaking mass splittings within the $\mathrm{SU}(5)$ representation, $M_{i} \neq M_{j}$.

$\dagger$ The combination $\delta_{\text {light }}$ proposed by A.E. Faraggi and B. Grinstein ( SSCL-Preprint-496, WIS-93/61 / JULY-PH, August, 1993) in their eqn. (3.14) is not ultraviolet finite for arbitrary matter representations of $\mathrm{SU}(5)$; their relation (3.13) between the three low energy gauge couplings is incorrect. Even if this ultraviolet divergence is ignored, taking their result seriously for $q^{2}=-M_{Z}^{2}$ would predict that top quark non-decoupling $\sim m_{\text {top }}^{2}$, that the relation between the three gauge couplings depends on neutral color singlet Higgs'and higgsinos, sneutrinos and weak neutral gauginos, and that the relation between gauge couplings would be affected by a new degenerate quark and lepton generation of mass $m \gg M_{Z}$; all of these predictions, which follow from the appearance in their formulae of oblique loops involving more than one spontaneously broken current, are incorrect. Their expression $\delta \sin ^{2} \theta$ in eqn. (3.11), which enters the relation between the two low energy electroweak gauge couplings, is also incorrect; both this and $\delta_{\text {light }}$ disagree with the results of the renormalization group "run and match" analysis ${ }^{[3][3][4]}$ of the broken $S U(5) \rightarrow S U(3)_{Q C D} \times U(1)_{Q E D}$ theory in the appropriate limits. Further, because they impose three mutually inconsistent conditions $(3.8,3.9,3.10)$ on gauge couplings, their $\mathrm{SU}(5)$ quantum field theory is mathematically self-inconsistent. 
The ultraviolet divergences in (4)and (3)cancel only in the grand unified theory; they will not cancel for arbitrary matter representations of $S U(3) \times S U(2) \times U(1)$. It follows that low energy high precision measurements can give information about grand unified matter representations, even for new states at the superheavy grand unified scale $M_{G U T}{ }^{[4]}$. This is very important for the study of threshold effects; $\mathrm{SU}(5)$ matter representations in which there is mass splitting between states do not, in certain circumstances, decouple from the relation between the three low energy gauge couplings at a given $q^{2}$ if the split states are electrically charged or color non-singlets. It is important to remember that, because (3)and (4)give a relation between gauge couplings, rather than dimensionful quantities arising from spontaneous symmetry breaking, any potential non-decoupling can increase at most $\sim \ln \frac{M_{i}}{M_{j}}$, rather than as powers $\sim \frac{M_{i}^{2}-M_{j}^{2}}{M_{Z}^{2}}$ as $\frac{M_{i}}{M_{z}} \rightarrow \infty$. (This is in contrast with the global $S U(2)_{L+R}$ breaking $\rho$ parameter ${ }^{[8]}$ which relates dimensionful quantities such as the precise $W$ and $Z$ masses.) In consequence, the non-decoupling of superheavy states in (4)is entirely defined by the ultraviolet divergence structure of the theory and therefore (since gauge couplings are at most logarithmically divergent) is completely logarithmic; there are no polynomial threshold corrections from superheavy matter states to relations between low energy gauge couplings in either supersymmetric or non-supersymmetric grand unified SU(5). This is illustrated by rederiving, in the formalism of this paper, the results of reference ${ }^{[4]}$. They identify three classes of superheavy states which enter minimal supersymmetric SU(5) gauge boson supermultiplets $V$, heavy components $H$ of the SU(5) chiral multiplet in which the Higgs' doublets lie, and the remnants $\Sigma$ of the superheavy particles which induced the breaking $S U(5) \rightarrow S U(3) \times S U(2) \times U(1)$ - and assign particles within a class a common mass $M_{V}, M_{H}$ or $M_{\Sigma}$. A simple calculation including all $\log$ and polynomial threshold effects gives

$$
\Delta_{S U_{5}}\left(-M_{Z}^{2}\right)_{\text {superheavy }}=\frac{2}{\pi} \ln \frac{M_{V}}{M_{\Sigma}}-\frac{6}{\pi} \ln \frac{M_{V}}{M_{H}}+\mathcal{O}\left(\frac{M_{Z}^{2}}{M_{V}^{2}}, \frac{M_{Z}^{2}}{M_{\Sigma}^{2}}, \frac{M_{Z}^{2}}{M_{H}^{2}}\right)
$$

Note the absence of any polynomials $\sim \frac{M_{i}^{2}-M_{j}^{2}}{M_{k}^{2}}$ with $i, j, k$ from the superheavy sector beyond those from expansion of renormalization group logs. 
There is another important consequence, for non-decoupling, of the presence of at least one exactly conserved $S U(3)_{Q C D} \times U(1)_{Q E D}$ current in the oblique vector self energies appropriate to relations between the gauge couplings. It has been known for some time that heavy degenerate chiral fermions with axial vector couplings do not necessarily decouple from low energy electroweak processes ${ }^{[9][6][5]}$ . A global $S U(2)_{L+R}$ conserving non-decoupling contribution from heavy degenerate fermions was identified ${ }^{[6][5][10]}$ and classified as affecting only relations between electroweak observables which have become dimensionful as a result of spontaneous symmetry breaking ${ }^{[5]}$. That particular non-decoupling effect does not occur in grand unified relations between dimensionless low energy gauge couplings. For example, $\Delta_{S U_{5}}$ is uninfluenced by the addition of a degenerate fourth generation of quarks and leptons, all of whose members have a common mass $m$, even if $m \sim M_{Z}$. Indeed, $\Delta_{S U_{5}}$ is independent of degenerate $\mathrm{SU}(5)$ matter representations, of either spin 0 or spin $\frac{1}{2}$, of any mass.

My attention now turns to threshold corrections from low mass states with masses $m<10 \mathrm{TeV}$. Here, both logs and polynomials will appear, depending on the experimental momentum transfer $q^{2}$. The simplest example is the effect of the three known generations of quarks and leptons. Because, in the absence of Majorana masses, the left-handed top quark $t$ and right-handed top quark $t_{c}$ are degenerate, and because they appear in the same 10 representation of $\mathrm{SU}(5)$ and cancel each other's contribution, $\Delta_{S U_{5}}$ does not depend on $m_{t o p}$ at all; the top quark is unable to affect relations between the three low energy gauge couplings even if $m_{\text {top }}^{2} \sim\left|q^{2}\right|$. Relation (4)receives only negligible $\sim \frac{m_{b}^{2}}{M_{Z}^{2}}$ threshold corrections from the three known quark and lepton generations on the $Z$ resonance where $-q^{2}=M_{Z}^{2} \gg m_{b}^{2}$. On the other hand, because the $b$ quark is split from its $\nu_{\tau}$ and $\tau$ partners in the $\underline{10}$ and $\underline{\overline{5}}$, there are threshold corrections for lower $\left|q^{2}\right| \sim m_{b}^{2}$

$$
\Delta_{S U_{5}}\left(\left|q^{2}\right| \sim m_{b}^{2}\right)=\frac{2}{\pi} \Re\left\{B_{3}\left(q^{2}, m_{b}, m_{b}\right)-B_{3}\left(q^{2}, 0,0\right)\right\}
$$

This integral has, of course, a rich and complicated log and polynomial structure ${ }^{[7]}$ 
; for $q^{2}=\frac{1}{4} m_{b}^{2},-4 m_{b}^{2}$, and $-M_{Z}^{2}$ it gives $\Delta_{S U_{5}} \approx+.2,-.25$ and $\mathcal{O}\left(\frac{m_{b}^{2}}{M_{Z}^{2}}\right)$ respectively. It serves as a first simple example of combination $\log$ and polynomial threshold behavior. Another example lies in corrections to the results of reference ${ }^{[4]}$ from low mass supersymmetric states. Imagine that the low mass particles have a complicated and interesting split spectrum, $m_{i}$ for the $i^{t h}$ low mass electrically charged and/or color non-singlet state, where some of the states are not too much heavier (and some might even be lighter) than $M_{Z}$; these splittings will induce polynomial threshold effects $\sim \frac{m_{i}^{2}}{M_{Z}^{2}}$ as well as logs. It is beyond the scope of this paper to investigate the detailed polynomial threshold corrections to relations between low energy gauge couplings in any specific supersymmetric model ${ }^{[1]}$. Still, a certain subclass of supersymmetric $\mathrm{SU}(5)$ models $^{[2]}$ generically contains such light states and they could contribute polynomial threshold effects to (4). A simple example is in order. Assume for simplicity that the squark and slepton superpartners of the three known generations of quarks and leptons all have a common mass $m_{S U S Y}$ except the superpartner $\tilde{t}$ of the left-handed top quark and the superpartner $\tilde{t}_{c}$ of the right-handed top quark; these I take to be eigenstates. Now calculate the squark and slepton contribution to $\Delta_{S U_{5}}$. Because of the mass degeneracy, all three $\underline{\overline{5}}$ generations cancel as do the $\underline{10}$ 's corresponding to the first two generations. Within the third generation $\underline{10}$, the $\tilde{b}$ and $\tilde{\tau}_{c}$ components cancel each other even if $m_{S U S Y}^{2} \sim\left|q^{2}\right|$. Because the $\tilde{t}$ and $\tilde{t}_{c}$ lie within the same $\underline{10}$ and are split, there remains

$$
\Delta_{S U_{5}}\left(q^{2}\right)=\frac{10}{\pi} \Re\left\{B_{13}\left(q^{2}, m_{\tilde{t}}, m_{\tilde{t}}\right)-B_{13}\left(q^{2}, m_{\tilde{t}_{c}}, m_{\tilde{t}_{c}}\right)\right\}
$$

The integral $B_{13}$ too has a rich and complicated $\log$ and polynomial structure ${ }^{[7]}$ for $m_{\tilde{\boldsymbol{t}}}^{2}$ and/or $m_{\tilde{\boldsymbol{t}}_{\mathrm{c}}}^{2} \sim\left|q^{2}\right|$ so the relation (4) on the $Z$ resonance will receive polynomial threshold corrections from the $\tilde{t}-\tilde{t}_{c}$ splitting. Non-decoupling for light states is also just logarithmic; as both $\tilde{t}$ and $\tilde{t}_{c}$ become heavy, $\Delta_{S U_{5}} \rightarrow \frac{5}{3 \pi} \ln \frac{m_{\tilde{i}}}{m_{\tilde{t}_{c}}}$. Although the contributions (7)and (8)are probably not experimentally observable, the point of principle remains intact; splittings within light SU(5) representations can contribute polynomial as well as logarithmic threshold effects to the $\mathrm{SU}(5)$ relation 
between the three low energy effective gauge couplings.

The presence of at least one current from the unbroken subgroup $S U(3)_{Q C D} \times$ $U(1)_{Q E D}$ in the appropriate oblique corrections to relations between low energy gauge couplings has another strong consequence; relations between any two or three low energy gauge couplings in supersymmetric or non-supersymmetric $\mathrm{SU}(5)$ depend only on electrically charged and/or color nonsinglet matter fields and are independent of colorless neutral matter fields. In particular, such relations do not depend on the standard model Higgs'. Neither do they depend on other neutral color singlet Higgs' or higgsinos, sneutrinos or neutral color singlet gauginos, such as weak zinos or photinos.

In addition to the above relation (4)between the three low energy gauge couplings, there exist in $\mathrm{SU}(5)$, of course, relations between any two gauge couplings. At leading $\log$, the price is that such relations will certainly depend on the superheavy gauge boson masses $\sim \ln \frac{M_{X}}{M_{W}}$ even if all superheavy matter representations are degenerate ${ }^{[]^{[3]}}$ with further dependence on the details of the superheavy spectrum if they are not ${ }^{[4]}$. The derivation of these relations to include all oblique one loop threshold effects of matter fields ${ }^{[12]}$ is as before; in order to obtain a relation between $g_{1}^{*}$ and $g_{2}^{*}$ simply rewrite the exact equation $\frac{1}{\left(g_{1}^{\text {bare }}\right)^{2}}-\frac{5}{3} \frac{1}{\left(g_{2}^{\text {bare }}\right)^{2}}=0$ in terms of the effective gauge couplings in the improved Born approximation at momentum transfer $q^{2}$ using (1):

$$
\left[\left\{\frac{1}{\left(g_{1}^{*}\right)^{2}}+\Re \Pi_{Y Q}^{\prime}\right\}-\frac{5}{3}\left\{\frac{1}{\left(g_{2}^{*}\right)^{2}}+\Re \Pi_{3 Q}^{\prime}\right\}\right]_{q^{2}}=0
$$

All oblique one-loop information about matter fields which can be obtained from the relation between the $S U(2)_{\vec{I}}$ and $U(1)_{Y}$ gauge couplings is therefore contained in a single function $\widetilde{\Delta}_{S U_{5}}$ if I rewrite (9)in the equivalent form ${ }^{[12]}$

$$
\begin{aligned}
& \frac{3}{8}-s_{*}^{2}-\frac{\alpha_{Q E D}^{*}}{4} \widetilde{\Delta}_{S U_{5}}=0 \\
& \widetilde{\Delta}_{S U_{5}}=16 \pi \Re\left\{\Pi_{3 Q}^{\prime}-\frac{3}{8} \Pi_{Q Q}^{\prime}\right\}
\end{aligned}
$$

where, as usual, everything is evaluated at the same $q^{2}$. 
Beyond its $\ln \frac{M_{X}}{M_{W}}$ dependence, the non-decoupling and threshold properties of $\widetilde{\Delta}_{S U_{5}}$ resemble closely those of $\Delta_{S U_{5}}$. The running of (10), analogous with equation (5), from one low $q^{2}$ to another is independent of all heavy matter states, split or degenerate. $\widetilde{\Delta}_{S U_{5}}$ does not depend on neutral states (and, in particular, it does not depend on the standard model Higgs'), is independent of degenerate SU(5) matter representations of either spin 0 or spin $\frac{1}{2}$, and has only logarithmic non-decoupling dependence on split superheavy states. It also does not depend on $m_{\text {top }}$. Therefore, neither the top quark or the standard model Higgs' are able to influence the SU(5) relation between the two low energy electroweak gauge couplings $\alpha_{Q E D}^{*}$ and $s_{*}^{2}$ or the relation between the three low energy effective gauge couplings $\alpha_{Q E D}^{*}, s_{*}^{2}$ and $\alpha_{Q C D}^{*} . \tilde{\Delta}_{S U_{5}}\left(q^{2}=-M_{Z}^{2}\right)$ is similarly unaffected by the three known quark and lepton generations while both log and polynomial threshold effects occur for lower $\left|q^{2}\right| ; \widetilde{\Delta}_{S U_{5}}\left(\left|q^{2}\right| \sim m_{b}^{2}\right)=\frac{1}{\pi} \Re\left\{B_{3}\left(q^{2}, m_{b}, m_{b}\right)-B_{3}\left(q^{2}, 0,0\right)\right\}$. Within the simple supersymmetric $\mathrm{SU}(5)$ model studied in equation (8), the same sorts of cancellations occur leaving $\widetilde{\Delta}_{S U_{s}}\left(q^{2}\right)=-\frac{2}{\pi} \Re\left\{B_{13}\left(q^{2}, m_{\tilde{t}}, m_{\tilde{t}}\right)-B_{13}\left(q^{2}, m_{\tilde{t}_{c}}, m_{\tilde{t}_{c}}\right)\right\}$ with a rich and complicated log and polynomial structure for $m_{\tilde{t}}^{2}$ and/or $m_{\tilde{t}_{c}}^{2} \sim\left|q^{2}\right|$; the relation (10)on the $Z$ resonance will therefore receive both log and polynomial threshold corrections from the $\tilde{t}-\tilde{t}_{c}$ splitting.

Comparison of the predictions of SU(5) with measurements of $s_{*}^{2}\left(q^{2} \approx 0\right)$ (from $e^{-} \nu$ scattering or, more speculatively, parity non-conservation in hydrogen) serves to illustrate the power of (10)over renormalization group techniques; it involves hadronic resonances and multi-hadron states and is properly described by neither logs or polynomials. Since (because of causality) the ח's are analytic functions

$$
\begin{aligned}
\tilde{\Delta}_{S U_{5}}\left(q^{2}=0\right) & =\tilde{\Delta}_{S U_{5}}\left(q^{2}\right) \\
& -16 q^{2} \mathcal{P} \int_{-\infty}^{0} \frac{d\left(q^{\prime}\right)^{2}}{\left(q^{\prime}\right)^{2}\left[\left(q^{\prime}\right)^{2}-q^{2}\right]} \Im\left\{\Pi_{3 Q}^{\prime}\left[\left(q^{\prime}\right)^{2}\right]-\frac{3}{8} \Pi_{Q Q}^{\prime}\left[\left(q^{\prime}\right)^{2}\right]\right\}
\end{aligned}
$$

where $\mathcal{P}$ indicates the principle part. The point $q^{2}$ is chosen so that $\widetilde{\Delta}_{S U_{5}}\left(q^{2}\right)$ is calculable in perturbation theory; the properly subtracted dispersion integral (11)includes a sum over experimental $e^{+} e^{-} \rightarrow$ hadrons data separated in isospin ${ }^{[13]}$. The 
lesson is clear; although precise threshold effects can be quite complicated, they are still given, when calculable, by (4)and (10). Of course, hadronic threshold effects are incalculable in $\Delta_{S U_{5}}\left(q^{2} \approx 0\right)$.

In Figure 1, $s_{*}^{2}\left(q^{2}=-M_{Z}^{2}\right)$ is displayed in minimal SU(5) ${ }^{[12]}$. Here the complete standard model results for the $\Pi$ 's are used and so automatically include all standard model threshold effects in $\widetilde{\Delta}_{S U_{5}}$. Quark and lepton masses are taken at their experimental values with $m_{\text {top }}=150 \mathrm{GeV} \cdot \alpha_{Q E D}^{*}\left(q^{2}=0\right)=\frac{1}{137.036}$ is used as experimental input with the usual hadronic dispersion relation ${ }^{[13]}$, including all threshold effects such as the appearance of the $\rho, \omega, \phi$ and $J / \Psi$ resonances as well as multi-hadron states, included in the running up to $\alpha_{Q E D}^{*}\left(q^{2}=-M_{Z}^{2}\right)=\frac{1}{128.658}$. Remember that the running of the electromagnetic coupling involves the combination $\Delta_{U(1)_{Q E D}}=16 \pi \Re\left\{\Pi_{Q Q}^{\prime}\left(q^{2}\right)-\Pi_{Q Q}^{\prime}(0)\right\}$ (where $\Pi_{Q Q}^{\prime}=\Pi_{Y Q}^{\prime}+\Pi_{3 Q}^{\prime}$ ) which, protected by the exact global $U(1)_{Q E D}$ charge symmetry, satisfies the usual decoupling theorem. The resulting experimental uncertainty from the dispersion relation $^{[13]} \delta_{\text {hadronic }} s_{*}^{2}= \pm .0003$ appears as the thickening of the lines in Figure 1. The standard model contribution of $W$ bosons is also included in the loops. For simplicity, all superheavy states are given a common mass $M_{X}$; it is the pole mass of the superheavy states, rather than an ambiguously defined grand unified scale, which enters (10). The non-observation of proton decay in the channel $p \rightarrow e^{+} \pi^{0}$ implies (after renormalization of the appropriate operator) a lower limit $M_{X} \geq 1.6 \times 10^{15} \mathrm{GeV}$; this is plotted as the vertical line in Figure 1. When $s_{*}^{2}\left(-M_{Z}^{2}\right)$ is plotted against $M_{X}$, the qualitative result is, of course, famous ${ }^{[1]} ;$ the experimental value $s_{*}^{2}\left(-M_{Z}^{2}\right)=.2313 \pm .001$ together with the limit from proton decay imply that minimal $\mathrm{SU}(5)$ is ruled out at the $>5 \sigma$ level.

Figure 1 also displays $s_{*}^{2}\left(-M_{Z}^{2}\right)$ from a supersymmetric $\mathrm{SU}(5)$ theory ${ }^{[12]}$. For pedagogical reasons, a particularly simple particle spectrum is chosen; all charged and/or color non-singlet superheavy states have a common mass $M_{X}, R$ parity $R \neq 0$ states corresponding to superpartners of states in the standard model have a common mass $\mu$ as do the extra charged Higgs' and their superpartners. Also displayed in Figure 1 are results ${ }^{[2]}$ for the simplest supersymmetric $E_{6}$ model, 
which includes an additional $U(1)$ and neutral $Z^{\prime}$ which cannot contribute to $\widetilde{\Delta}_{S U_{5}}$ for $\frac{M_{Z}^{2}}{M_{Z^{\prime}}^{2}} \ll 1$. Again, the qualitative result of Figure 1 is famous ${ }^{[1]}$; supersymmetric grand unified theories predict higher $s_{*}^{2}\left(-M_{Z}^{2}\right)$ and higher $M_{X}$ while avoiding conflict with nucleon decay experiments.

The lesson of Figure 1, equation (6)and reference ${ }^{[4]}$ is that relations between gauge couplings in grand unified theories depend on the details of the superheavy spectrum. Beyond issues of personal taste, the spectrum of masses $M>10^{8} \mathrm{GeV}$ can be arbitrarily complicated and generic predictive power of (4)and (10)is lost. Add to this the issue of $\log$ and polynomial threshold effects from the detailed low mass $m<10 \mathrm{TeV}$ spectrum in supersymmetric models ${ }^{[1]}$ and it is clear that, for arbitrary soft supersymmetry breaking sectors, supersymmetric grand unified models contain enough parameters to saturate almost any high precision measurement of the relation between any two or three low energy gauge couplings. The result is quite worrisome; all generic low energy high precision predictive power of supersymmetric grand unified theories for relations between gauge couplings has been lost. Most distressing is that the study of high precision relations between gauge couplings is certainly unable to generically disentangle the contributions of superheavy states from those of the low mass superpartner sector ${ }^{[14]}$.

Recently, a certain subclass of minimal supersymmetric $\mathrm{SU}(5)$ models $^{[2]}$ has come under close scrutiny because they "naturally" impose strong constraints on the soft supersymmetry breaking terms at the grand unified scale $M_{G U T}$ and also have a highly constrained pattern of fermion masses and mixings. Further, their superpotential imposes strong constraints on the superheavy mass spectrum and, therefore, its logarithmic threshold effects. Most interesting, though, is that this subclass generically contains light states $m_{\text {light }} \sim M_{Z}$. Threshold effects of such particles cannot be analyzed with the leading log behavior of the renormalization group. Rather, they are precisely given by equations (4)and (10). It is beyond the scope of this paper to examine log and polynomial threshold effects for any specific grand unified model; the interest of this paper lies in pointing out the origin and generic existence of such effects, along with the generic character of any potential 
non-decoupling. Still, threshold effects in this class of minimal supersymmetric models, which have an interesting non-degenerate light particle spectrum, may give important corrections to relations between the effective low energy gauge couplings measured in high precision experiments via (4)and (10). On the other hand, since strong constraints are imposed on relations between gauge couplings by the necessary appearance of exactly conserved $S U(3)_{Q C D} \times U(1)_{Q E D}$ currents, this class of theories may give only negligible contributions to $\Delta_{S U_{5}}$ and $\widetilde{\Delta}_{S U_{5}}$ for most of its parameter space; in that case, high precision experimental determinations of low energy gauge couplings may give strong generic constraints on supersymmetric $\mathrm{SU}(5)$ theories.

The philosophy of this paper, which follows closely that of reference ${ }^{[5]}$, differs dramatically from that of the usual "run and match" renormalization group anal$\operatorname{ysis}^{[1][3][4]}$. It is also far more powerful for the purposes of comparison between low energy high precision observables. No mention has been made here of any renormalization scale $\mu^{2}$ or of operators evaluated at superhigh energies $E \sim M_{X}$; neither do I refer to ambiguously defined quantities such as a grand unification scale $M_{G U T}$, subgroup gauge couplings $g_{1}\left(M_{G U T}\right), g_{2}\left(M_{G U T}\right), g_{3}\left(M_{G U T}\right)$ evaluated there, or even of a grand unified gauge coupling $g_{5}\left(M_{G U T}\right)$ evaluated there. Instead, relations between low energy high precision observables are shown to follow simply from the symmetries and dynamical Ward identities of the bare $S U(5)$ Lagrangian as well as those of its subset, the bare $S U(3)_{Q C D} \times S U(2)_{\vec{I}} \times U(1)_{Y}$ Lagrangian. Low energy $\mathrm{SU}(5)$ calculations are done on their own terms here. Of course, the results of the formalism presented in this paper agree with the results of the renormalization group run and match analysis of the broken $S U(5) \rightarrow$ $S U(3)_{Q C D} \times U(1)_{Q E D}$ theory when the mass spectra of new states is very heavy compared to the experimental momentum transfer; in that case, both new light states $\left(\left|q^{2}\right| \ll m_{i}^{2}<100 T e V^{2}\right)$ and superheavy states will contribute only logs, in analogy with equation (6). For much lighter states $\left(m_{j}^{2} \sim\left|q^{2}\right|\right)$, the renormalization group analysis is, of course, inadequate, and the full equations (4)and (10)must be used. 
Although, for reasons of clarity and pedagogy, I have here concentrated on $\mathrm{SU}(5)$, all of the results of this paper apply to any larger grand unified gauge group $\mathcal{G}$, such as $S O(10)$, with $\mathcal{G} \supseteq S U(5) \supset S U(3)_{Q C D} \times S U(2)_{\vec{I}} \times U(1)_{Y}$. This is because the relations between bare and effective low energy gauge couplings (1)as well as the exact grand unified relations between bare gauge couplings (2)and $g_{2}^{\text {bare }}=g_{3}^{\text {bare }}$ still hold.

The results of this paper differ qualitatively from the previous renormalization group leading $\log ^{[3][4]}$ analysis. Two important points of principle are to be insisted upon: Firstly, the quantitative results from the expressions $\Delta_{S U_{5}}$ and $\widetilde{\Delta}_{S U_{5}}$ include all calculable oblique one loop low energy threshold contributions (logs, polynomials, hadronic resonances for $\widetilde{\Delta}_{S U_{5}}\left(q^{2} \approx 0\right)$, everything) to relations between any two or three low energy gauge couplings from $\mathrm{SU}(5)$ matter representations and are far more accurate than is possible using one loop renormalization group techniques. Two loop renormalization group effects can then be added by hand. Secondly, the technique of equations (1), (3)and (9)is able to identify with great accuracy which quantities are measured in high precision experiments and compare theoretical calculations with these; apples are compared to apples, oranges to oranges.

In this paper, I have generalized the leading log relations between low energy gauge couplings to include all oblique one loop threshold effects from matter fields in both supersymmetric and non-supersymmetric $\mathrm{SU}(5)$. In all cases the character of these threshold effects, and any potential non-decoupling, is deeply constrained by the appearance of exactly conserved currents from the unbroken $S U(3)_{Q C D} \times U(1)_{Q E D}$ subgroup. I have displayed the common origin of the logs due to split superheavy matter states, which can be found with renormalization group techniques, and the combinations of logs and polynomials (and, in the case of hadrons, resonant and multi-hadron terms) appropriate to light matter states, which cannot be found with renormalization group techniques. I have shown that oblique one loop $\mathrm{SU}(5)$ relations between any two or all three low energy effective gauge couplings do not depend on the top quark or standard model Higgs' masses. 
Such relations also do not depend on neutral color singlet states, such as other neutral color singlet Higgs, or, in supersymmetric $\mathrm{SU}(5)$, sneutrinos, zinos, photinos or neutral color singlet higgsinos. Neither do they depend on degenerate SU(5) matter representations, of either spin 0 or spin $\frac{1}{2}$, of any mass. The splitting between the $b$ quark and its $\mathrm{SU}(5)$ partners, the $\nu_{\tau}$ and $\tau$, generates both $\log$ and polynomial threshold effects for relations between gauge couplings at $\left|q^{2}\right| \sim m_{b}^{2}$. Splitting between new low mass states, such as occur in supersymmetric theories, can also generate both $\log$ and polynomial corrections. Certain minimal supersymmetric $\mathrm{SU}(5)$ theories, which control the spectrum of superheavy states while generically producing a complicated mass split spectrum of light states, may contribute important polynomial threshold effects as well as logs to relations between low energy gauge couplings, especially near the $Z$ resonance. For very heavy spectra $m_{i}^{2} \gg\left|q^{2}\right|$, the results of the formalism presented here agree with the results of the renormaliztion group "run and match" analysis in the broken $S U(5) \rightarrow S U(3)_{Q C D} \times U(1)_{Q E D}$ theory. All of the results of this paper (logs, polynomials, everything) also hold for any larger grand unified gauge group $\mathcal{G} \supseteq S U(5) \supset S U(3)_{Q C D} \times S U(2)_{\vec{I}} \times U(1)_{Y}$. With the techniques introduced here, high precision experimental results can be compared reliably with the theoretical predictions of grand unified theories.

\section{DISCLAIMER}

This report was prepared as an account of work sponsored by an agency of the United States Government. Neither the United States Government nor any agency thereof, nor any of their employees, makes any warranty, express or implied, or assumes any legal liability or responsibility for the accuracy, completeness, or usefulness of any information, apparatus, product, or process disclosed, or represents that its use would not infringe privately owned rights. Reference herein to any specific commercial product, process, or service by trade name, trademark, manufacturer, or otherwise does not necessarily constitute or imply its endorsement, recommendation, or favoring by the United States Government or any agency thereof. The views and opinions of authors expressed herein do not necessarily state or reflect those of the United States Government or any agency thereof. 


\section{ACKNOWLEDGEMENTS}

Some of the seminal ideas and results of this paper appeared long ago in work with my colleague Dallas C. Kennedy ${ }^{[12]}$; I thank him as I would a co-author. I thank J. Thomas for valuable physics discussions and opinions and help with the computational jungle. I thank F. Gilman and the members of the Theoretical Physics Division for their hospitality at the Superconducting Super Collider Laboratory during the academic year 1992-93. This work was supported in part by a National Science Foundation Grant NSF-PHY-89-17438, by the Universities Research Association with Department of Energy contract DE-AC35-89ER40486 and by a 1992-93 SSC Fellowship from the Texas National Research Laboratory Commission. 


\section{REFERENCES}

1. For a review, see G. Ross, Grand Unified Theories, Benjamin- Cummings, Menlo Park, Ca. (1984).

2. S. Martin and P. Ramond, NUB-3067-93TH, UFIFT-HEP-93-16, SSCLPreprint-439, June, 1993; J. Ellis and F. Zwirner, Nuc. Phys. B338 (1990) 317; S. Kelley, J. Lopez, H. Pois, D.V. Nanapoulos and K. Yuan, Phys. Letts. B273 (1991) 423; M. Drees and M.M. Nojiri, Nuc. Phys. B369 (1992) 54; P. Nath and R. Arnowitt, Phys. Rev. Letts. 69 (1992) 725; R. Arnowit and P. Nath, CTP-TAMU-52/93, NUB-TH-3073-93, SSCL-Preprint 503-93 (1993); D.J. Castano, E.J. Piard and P. Ramond, UFIFT-HEP-93-18 (1993); On a different tack, G.L. Kane, C. Kolda, L.Roszkowski and J.B. Wells, U. Michigan Preprint, UM-TH-93-24, (1993) have, without a specific choice of gauge group, considered a complete set of phenomenological contraints on grand unified supersymmetric theories so as to recover predictive power.

3. U. Amaldi, W. de Boer and H. Fustenau, Phys. Letts. B260 (1991) 447; P. Langacker and M. Luo, Phys. Rev. D44 (1991) 817; J. Ellis, S. Kelley and D.V. Nanopoulos, Phys Letts. B249 (1990) 441; B260 (1991) 131; B287 (1992) 95; Nuc. Phys B373 (1992) 75; H. Arason et. al. Phys. Rev. D46 (1992) 3945; F. Anselmo, L. Cifarelli, A. Peterman and A. Zichichi, Nov. Cim. A105 (1992) 1179; P. Langacker, Proc. PASCOS-90 Sym., P. Nath and S. Reucroft (editors), World Scientific, Singapore, 1990; G.G. Ross and R.G. Roberts, Nuc. Phys. B377 (1992) 571; P. Langacker and N. Polonski, Phys. Rev. D47 (1993) 4028; A. Giveon, L.J. Hall and U. Sarid, Phys. Letts. B271 (1991) 138 .

4. R. Barbieri and L.J. Hall, Phys. Rev. Letts. 68 (1989) 752; S. Meshkov, B. Grinstein and A.E. Faraggi, Phys. Rev. D47 (1993) 5018; K. Hagiwara and Y. Yamada, Phys. Rev. Letts. 70 (1993) 709.

5. D.C. Kennedy and B.W. Lynn, SLAC-Pub 4039 (1987); Nucl. Phys. B322 
(1989) 1; B. W. Lynn, Unitarity, Causality and High Precision Physics, Stanford Preprint SU-ITP-93-23, September, 1993.

6. B.W. Lynn, M.E. Peskin and R.G. Stuart in Physics at LEP, J. Ellis and R. Peccei (editors), CERN Report 86-02, QC770.E82.86-02 (1986), Vol. 1, pg. 90 , first identified this universal set of corrections, in the standard model or due to matter representations from beyond the standard model, as entering all massless four-fermion processes in the same way; the term "oblique" is due to V. Peskin.

7. The basic work is G. 't Hooft and M. Veltman, "Diagrammar", in Particle Interactions at Very High Energies, D. Speiser et. al. (editors), Plenum Press, New York, 1974; A complete discussion of all integrals appearing in one loop calculations, including their analytic properties, would combine R.G. Stuart, Comp. Phys. Comm. 48 (1988) 367 and R.G. Stuart and A. Gongora-Trevino. Comp. Phys. Comm. 56 (1990) 337 with G. 't Hooft and M. Veltman, Nuc. Phys. B153 (1979) 365 . The two point integrals $B_{3}=B_{21}+B_{1}$ and $B_{13}=B_{3}+\frac{1}{4} B_{0}$ appearing in this paper are defined in terms of integrals appearing there.

8. M. Veltman, Nuc. Phys. B123 (1977) 89; M. Einhorn, D. Jones and M. Veltman, Nuc. Phys. B191 (1981) 146; M. Chanowitz, M. Furman and I. Hinchliffe, Phys. Letts. B78 (1978) 285; Nuc. Phys. B153 (1979) 402.

9. M. Veltman, Nuc. Phys. B123 (1977) 89; S. Sarantakos and A. Sirlin, Nuc. Phys. B217 (1983) 84.

10. M.E. Peskin and T. Takeuchi, Phys. Letts. 65 (1990)964; Phys. Rev. D46 (1992) 381; G. Altarelli and R. Barbieri, Phys. Letts. B253 (1991) 161; D.C. Kennedy and P. Langacker, U. Penn. Preprint UPR-0436T (1990).

11. B. W. Lynn, SLAC-Pub-3358 (June, 1984) first suggested and showed that, for a low supersymmetry breaking scale $m_{\frac{3}{2}}$, quantum loop effects in supersymmetric $S U(2)_{\vec{I}} \times U(1)_{Y}$ gave measurable shifts in low energy high 
precision electroweak observables while, for a large $m_{\frac{3}{2}}$, they decoupled. Complete oblique one-loop formulae, including all threshold effects, are displayed there for arbitrary soft supersymmetry breaking sectors. The results, included in B.W. Lynn, M.E. Peskin and R.G. Stuart in Physics at LEP, J. Ellis and R. Peccei (editors), CERN-Report 86-02, QC770.E82.86-02 (1986), Vol 1. pg. 90, have from the beginning been part of the LEP/SLC program of high precision electroweak physics.; K.H.G. Schwarzer, OXFORD-TP 40/84 (September, 1984); B.W. Lynn in Polarization at LEP, G. Alexander et. al. (editors), CERN Report 88-06, QC770.E82.88-06 (1988), Vol 1, pg. 24; J. Ellis, S. Rudaz and N. Tracas, CERN-TH-4657/87 (1987); J.A. Grifols and J. Sola, Nuc. Phys. B253 (1985) 47; Phys. Letts. B137 (1984) 257; R. Barbieri and L. Maiani, Nuc. Phys. B224 (1983) 32; E. Eliasson, Phys. Letts. B147 (1984) 65; L. Alvarez-Guame, J. Polchinski and M. Wise, Nuc. Phys. B221 (1983) 495; C.S. Lim, T. Inami and N. Sakai, Phys. Rev. D29 (1984) 1488 ;

12. D.C. Kennedy and B.W. Lynn, SLAC-Pub-4608 (1988); B.W. Lynn in Polarization at LEP, G. Alexander et. al. (editors), CERN Report 88-06 (1988), QC770.E82.88-06 (1988), Vol. 1, pg. 24

13. H. Burkhardt, F. Jegerlehner, G. Penso and C. Verzegnassi, Z. Phys. C43 (1989) 497; B.W. Lynn, G. Penso and C. Verzegnassi, Phys. Rev. D35 (1987) 42.

14. The disentanglement, in supersymmetric grand unified theories, of quantum loop contributions to low energy electroweak observables due to superheavy $M>10^{8} \mathrm{GeV}$ states from those of light $m<10 \mathrm{TeV}$ states is found in B.W. Lynn, Supersymmetric $S U(3)_{Q C D} \times S U(2)_{\vec{I}} \times U(1)_{Y}$, Supersymmetric Grand Unified Theories and High Precision Electroweak Physics, Stanford Preprint SU-ITP-93-24, September, 1993. 


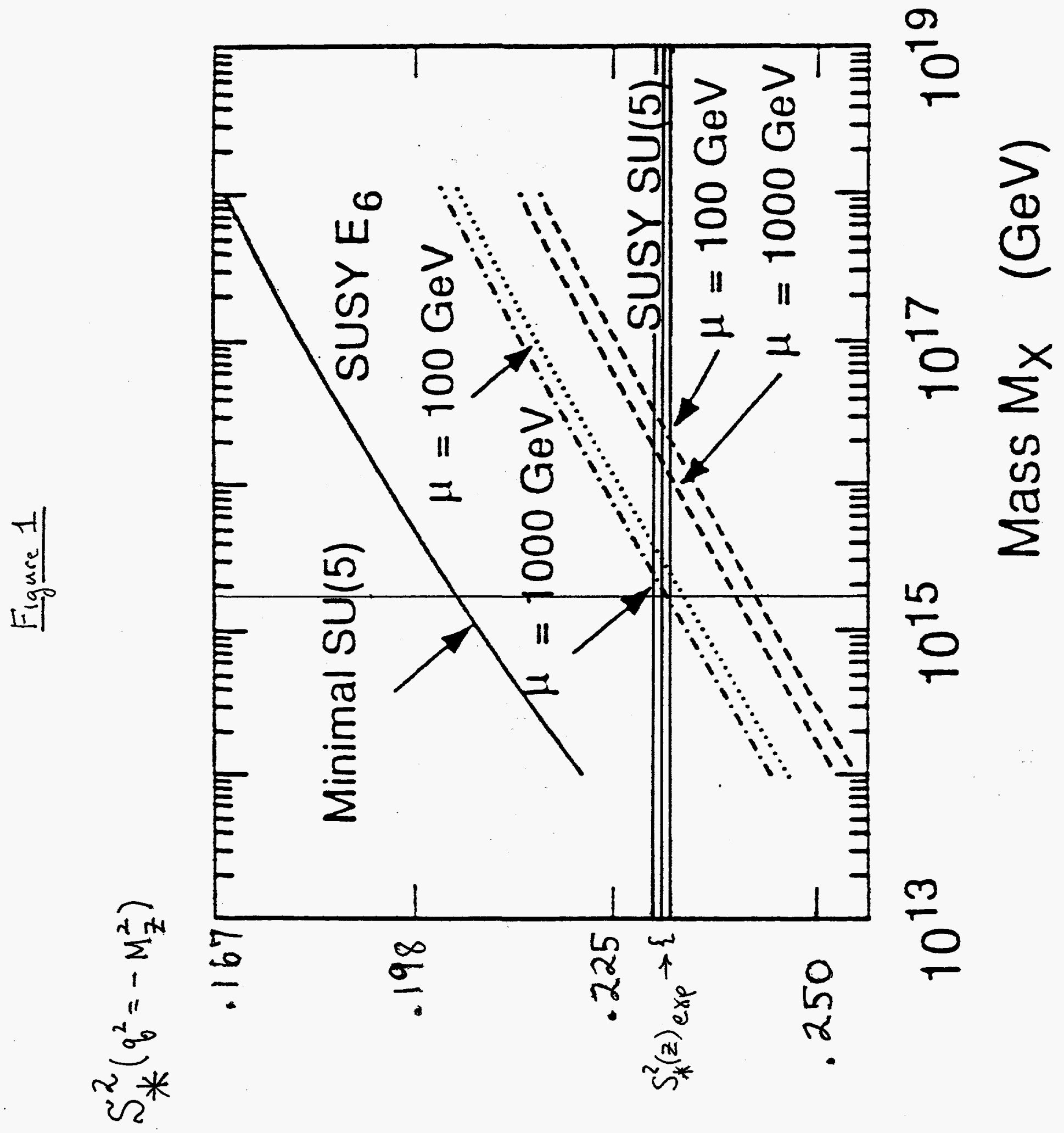

